\title{
Orientation Mapping by Electron Channeling (OMEC) and Hybrid Stage/Beam- Rocked Electron Channeling Patterns
}

\author{
Benjamin D. Myers ${ }^{1,2}$, Karl A. Hujsak ${ }^{2}$ and Vinayak P. Dravid ${ }^{1,2}$ \\ 1. NUANCE Center, Northwestern University, Evanston, IL. \\ 2. Department of Materials Science and Engineering, Northwestern University, Evanston, IL.
}

Orientation mapping of bulk, polycrystalline materials by electron backscatter diffraction (EBSD) has become an important and relatively pervasive analytical tool to link microstructure to material properties and performance. In contrast, techniques based on electron channeling such as selected area channeling patterns (SACP) and electron channeling contrast imaging (ECCI) have been relegated to mostly niche applications. The primary driver for this is the rapid development of EBSD over the past two decades to improve pattern acquisition speeds, indexing accuracy and general usability for orientation mapping and phase identification. This is largely driven by advances in computational capabilities, but similar advances have yet to find application in the generation of electron channeling patterns (ECP), as the acquisition of which has traditionally required specialized electron optics and provides limited spatial resolution and angular field-of-view. This is despite significant potential advantages of electron channeling techniques, including the direct visualization of dislocations and strain analysis via HOLZ lines. In this work, we present the development of a stage-rocked method for generating ECPs, which relies on automated imaging at a range of different sample orientations followed by computational image alignment, segmentation and ECP extraction.

The concept of a stage-rocked ECP was posited over 50 years ago [1], yet reference to an experimental realization of such a technique is difficult to find in literature. The primary challenge for a stage-rocking approach lies in the initial assumption that the beam should remain fixed, while the sample is manipulated by means of a eucentric goniometer stage. However, the precision of such a stage would need to be extremely high (even by today's standards) in order to achieve reasonable spatial resolution. We have circumvented this problem by recognizing that the stages in many commercial microscopes are sufficiently precise to achieve micron-scale precision in these types of operations and we apply image alignment algorithms to correct for any displacement. Briefly, the OMEC technique relies on automated image acquisition at a range of tilt/rotation conditions. The resulting images are aligned using a scale invariant feature transform (SIFT) algorithm, which aligns images and corrects for image misalignment, skew, rotation, etc [2]. This aligned image stack can then be processed to extract ECPs from any subset of pixels or segmented to extract ECPs from individual grains.

The raw ECPs generated by this technique contain as many pixels as there are sample orientations specified during data collection (typically on the order of $10^{4}$ ), which are projected on the surface of a sphere with no angular distortion. Figure 1a shows a stage-rocked ECP for a single crystal silicon (100) sample collected with 7,200 stage points. One interesting side-effect of the OMEC process is that the actual beam-sample orientation is modified at each pixel of a raster-scanned image by the beam divergence due to scanning. This is the same mechanism responsible for generation of ECPs by using low-magnification imaging of large crystals and effectively results in the collection of a beam-rocked ECP at every position in the stage-rocked ECP. By applying a beam divergence correction to each pixel at each stage position, the number of orientation points can be dramatically expanded (up to $10^{10}$ for a $1 \mathrm{~K} \times 1 \mathrm{~K}$ image with $10^{4}$ stage positions) and thereby significantly improving the angular resolution of the 
ECP. Figure $1 \mathrm{~b}$ shows the same silicon (100) data set with beam divergence correction applied. At each stage position, a $512 \times 442$ raw image was collected, which was then cropped and binned to a $16 \times 16$ pixel data set for a total of over 1.8 million data points. The utility of this approach will depend on the beam divergence for a given data set (grain size and image condition-dependent) and the degree of subsampling will depend on the signal-to-noise ratio in the raw images. Nevertheless, this approach allows an improvement to angular resolution of the stage-rocked ECPs and the OMEC technique itself offers the possibility of orientation mapping on a standard SEM with no specialized hardware. This contribution will introduce the OMEC technique and focus on the hybrid beam- and stage-rocked patterns that can be generated by the combination of raster-scanning with stage rocking [3].

References:

[1] D. G. Coates, Philosophical Magazine 16 (1967), p. 1179.

[2] D. G. Lowe, The proceedings of the seventh IEEE international conference on Computer Vision (1999) p. 1150.

[3] This material is based on research sponsored by the Air Force Research laboratory under agreement number is FA8650-15-2-5518. This work was also sponsored by the Air Force Office of Scientific Research under Award No. FA9550-12-1-0280. This work made use of the EPIC facility of Northwestern University's NUANCE Center, which has received support from the Soft and Hybrid Nanotechnology Experimental (SHyNE) Resource (NSF ECCS-1542205); the MRSEC program (NSF DMR-1720139) at the Materials Research Center; the International Institute for Nanotechnology (IIN); the Keck Foundation; and the State of Illinois, through the IIN.
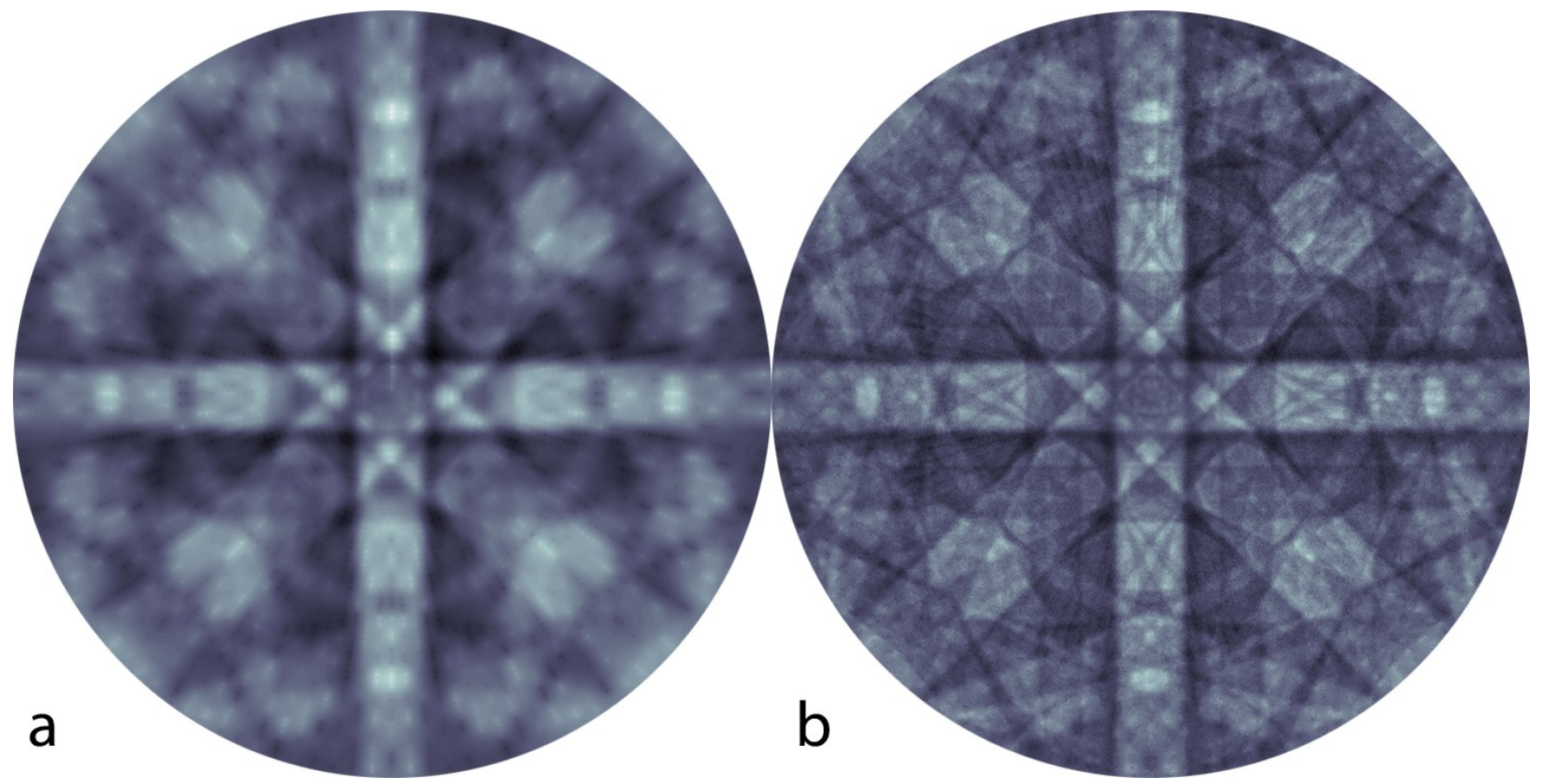

Figure 1. Electron channeling patterns of silicon (100) generated by (a) stage-rocking and inpainting and (b) stage-rocking with beam divergence correction, interpolation and inpainting. 